\title{
A Perdition Study on the Guangdong Province GDP Based on BP Neural Network
}

\author{
Huang Rui ${ }^{1}$, Tang Zilong ${ }^{1}$, Li Bin ${ }^{1,2}$ \\ 1. Behavioral Finance and Regional Laboratory of Guangdong \\ University of Finance \\ 2. Guangzhou Key Research Bases of Regional Financial Policy of \\ Guangdong University of Finance (Correspondence author)
}

\begin{abstract}
The changes of GDP continue to increase in China since 2007. Therefore, it is going to use the National Bureau of Statistics Data, which takes the application of BP neural network with momentum of the points, carries on the forecast to the Guangdong Province GDP. The conclusion shows that BP neural network can predict the Guangdong Province GDP healthy, and it is going to propose some policy suggestions.

Keywords: GDP; BP neural network; forecast; countermeasures
\end{abstract}

\section{Introduction}

It is obvious that the pace of economic development for a city or region is affected by many factors. But the two major aspects are the external factors, including location, natural endowment; and the internal factors, including the economic foundation, innovation ability and the choice of industry. By analyzing the evolution of all these two aspects, the GDP growth and its spatial distribution can be well explained, that is city spatial attribute data contains the location information in the critical external factors, while the total number of city GDP in enormous scale contains endogenous factors in its economic information. Therefore, based on perdition of GDP growth may explain the different levels of development to different city spatial structure, as well as can make an effective judgment on the development trend of similar individual city.

The gross domestic product (GDP) as an important indicator to reflect the 
level of economic development, and all its analysis and the formulation of monetary policy, price policy, consumption policy, wage policy and provide scientific are superior for the national economic accounting. Therefore, the real-time monitoring and prediction of Guangdong Province GDP is undoubtedly great significance to develop the next step of macroeconomic policy. It is surely that the prediction of Guangdong Province GDP depends on China's overall macroeconomic situation heavily. Generally speaking, the whole macro economic system is complex, and it is difficult to determine the nonlinear function if it used to describe the input and output characteristic of the whole system.

Moreover, psychologist W. S. McCulloch and mathematician W. Pitts proposed M-P model since 1943, which artificial neural network research has experienced from rise to depression. Nevertheless, BP neural network has a strong ability of approaching nonlinear function, which can consistent with the sample data of training, then designate a functional relationship between input and output variables, determine the coupling weights between neurons, so that the entire BP neural network has the approximate function. According to the National Bureau of statistics data which published on the website, through the application of a BP neural network with momentum term, and it is believe that it can be used to predict the Guangdong Province GDP lucratively.

\section{BP Neural Network}

BP neural network has the input layer, output layer, and hidden layer. But the simplest BP network is only one hidden layer. Within BP network, each layer of neurons can be different. And there are many connections between the previous layer and next layer, and the nonlinear transfer function of each neuron is type $S$ function. The most commonly used function form is $\varphi(x)=\frac{1}{1+e^{-\alpha x}}$, which the parameter $\alpha>0$ means it can control the slope.

BP learning algorithm has two phases. For one side is the computing forward from the input layer of the network, if the network weights and threshold of each layer has been set, then input the known samples, it can calculate the output of each layer of neurons. On the other side is the calculation backward from the last output layer, it changes the weights between each layer and each layer of threshold gradually. It is suggested that set these two stages of the process repeatedly, until the error is less than the setting value, or select the minimum error weights and thresholds in the setting of learning cycle.

The input vector in the $\mathrm{h}$ period of training sample is $x(h)=\left(x_{1}(h), x_{2}(h), \ldots, x_{v}(h)\right)$, where $v$ is the number of input layer neurons, the desired objective vector network output is $t(h)=\left(t_{1}(h), t_{2}(h), \ldots, t_{v}(h)\right)$, there are $\mathrm{K}$ hidden layers totally. The output vector $\mathrm{j}$ layer is $t^{(j)}(h)=\left(t_{1}^{j}(h), t_{1}^{j}, \ldots, t_{v z}{ }^{j}(h)\right)$, where $v_{z}$ is the number of the 
z layer in the neural network. $w_{j i}^{(j+1)}(h)$ is the weights connected to the $\mathrm{j}$ layer to the hidden layer of the $\mathrm{i}$ neurons and $\mathrm{j}$ neurons. Threshold $p_{j}^{(j+1)}(h)$ is the $\mathrm{j}$ neurons in hidden layer which corresponding with $\mathrm{j}+1$ layer. Set $t^{(0)}(h)=x(h)$, $t^{(j+1)}(h)=t(h) \quad$, then each neuron output can meet: $t_{i}^{(j+1)}(h)=\varphi\left(\sum_{i=1}^{P_{j}} w_{j i}^{j+1}(h) y_{i}^{j}(h)-p_{i}^{(j)}(h)\right)(0 \leq j \leq k)$ if

$$
w_{i 0}^{(j+1)}(h)=p_{i}^{(j+1)}(h), t_{0}^{j}(h)=-1
$$

$\operatorname{so} t_{i}^{j+1}(h)=\varphi\left(\sum_{i=0}^{j+1} w_{j i}^{j+1}(h) t_{i}^{j}(h)\right)(0 \leq j \leq k)$. If $b_{i}^{j+1}(h)=\sum_{i=0}^{P_{k}} w_{j i}^{j+1}(h) t_{i}^{j}(h)$, thus $t_{i}^{(j+1)}(h)=\varphi\left(b_{i}^{j+1}(h)\right)(0 \leq j \leq k)$. If define the error and square error of $\mathrm{m}$ neurons in network layer respectively: $e_{m}(h)=d_{m}(h)-t_{m}(h), E_{m}(h)=e_{m}^{2}(h)$, the total squared error for output layer (P neurons) is $E(h)=\sum_{i=1}^{P} E_{i}(h)=\sum_{i=1}^{P} e_{i}^{2}(h)$, and the total squared error of $\mathrm{N}$ periodic sample goes to $E=\sum_{i=1}^{N} E(h)$.

In practical application, it is very important to choose the learning rate of $\alpha(h)$, if $\alpha(h)$ goes convergence fast, it's large may cause instability; otherwise, $\alpha(h)$ can avoid the oscillation if the speed of convergence is slow. The simplest way to solve this contradiction is to join the momentum and the rate of adaptive learning. Therefore, $\Delta w_{j i}(h)=\beta \Delta w_{j i}(h-1)+\alpha(h) \delta_{j}(h) y_{j}(h)$, where $0<\beta<1$, which is the second correction of conventional BP algorithm, the first one is the momentum, $\alpha$ is the momentum coefficient, adaptive learning rate for $\alpha(h)$ is:

$$
\alpha(n)=\left\{\begin{array}{c}
\gamma \alpha(h-1), \text { if } E(h)<E(h-1) \\
\beta \alpha(h-1), \text { if } E(h) \geq k E(h-1) \\
\gamma \alpha(h-1), \text { other }
\end{array}\right.
$$

The typical parameter values $\beta=1.05, \gamma=0.7, k=1.04$. 


\section{The empirical analysis}

These papers takes Guangdong Province as an example, using the GDP as the input vector from 2007 to 2010, and utilize the GDP in 2011 as the target vector. It is going to instruct the BP neural network, to verify whether it can meet the need of prediction (All data from Guangdong Statistical Yearbook).

Generally speaking, it usually applies the available time series data for training and testing data. According to the network structure composed of the parameters of the input output model, each layer of the programming for the activation function is $\varphi(x)$, the output layer neuron number is 2 , when the number of the input neurons goes to $\mathrm{J},\left(x_{t}, x_{t+1}, \ldots, x_{t+j}\right)$ is the input vector $\mathrm{t}$, so it can obtain $\left(\frac{1}{1+e^{\left(-x_{k+s}\right)}}, \frac{1}{1+e^{\left(-x_{k+s+1}\right)}}\right)$. In the prediction process, when the network output is $\mathrm{y}$, the forecast value is $\log \left(\frac{y}{1-y}\right)$. Therefore, it is going to predict the 21 cities' GDP in Guangdong Province, using the matlab to write the BP neural network program. Setting the network hidden layer has 21 nodes, the max training is 3000 steps, the training objective minimum error is 0.001 , every 100 steps can acquire a training result, and the learning rate is 0.05 .

Table 1: Guangdong GDP from 2007 to 2011

\begin{tabular}{|l|l|l|l|l|l|}
\hline Cities & 2007 & 2008 & 2009 & 2010 & 2011 \\
\hline Guangzhou & 7140.32 & 8287.38 & 9138.21 & 10748.28 & 12423.44 \\
\hline Shenzhen & 6801.57 & 7786.79 & 8201.32 & 9581.51 & 11505.53 \\
\hline Zhuhai & 894.81 & 997.16 & 1038.66 & 1208.60 & 1404.93 \\
\hline Shantou & 829.49 & 951.81 & 1035.87 & 1208.97 & 1275.74 \\
\hline Foshan & 3660.18 & 4378.30 & 4820.90 & 5651.52 & 6210.23 \\
\hline Shaoguan & 481.14 & 553.85 & 578.75 & 683.10 & 816.81 \\
\hline Heyuan & 329.61 & 395.88 & 405.50 & 475.14 & 579.29 \\
\hline Meizhou & 411.62 & 479.61 & 519.29 & 612.85 & 707.54 \\
\hline Huizhou & 1117.91 & 1304.05 & 1414.70 & 1729.95 & 2093.08 \\
\hline Shanwei & 288.71 & 346.58 & 390.04 & 465.08 & 550.55 \\
\hline Dongguan & 3160.05 & 3703.60 & 3763.91 & 4246.45 & 4735.39 \\
\hline Zhongshan & 1268.04 & 1457.01 & 1566.41 & 1850.65 & 2193.20 \\
\hline Jiangmen & 1097.26 & 1270.88 & 1340.88 & 1570.42 & 1830.64 \\
\hline Yangjiang & 405.29 & 480.50 & 527.27 & 639.84 & 766.82 \\
\hline Zhanjiang & 924.41 & 1099.41 & 1156.67 & 1405.06 & 1700.23 \\
\hline Maoming & 997.08 & 1177.84 & 1231.25 & 1492.09 & 1745.31 \\
\hline Zhaoqing & 619.69 & 760.50 & 862.00 & 1085.87 & 1324.41 \\
\hline
\end{tabular}




\begin{tabular}{|l|l|l|l|l|l|} 
Qingyuan & 557.66 & 652.10 & 715.14 & 869.79 & 1003.03 \\
\hline Chaozhou & 372.80 & 438.08 & 480.18 & 559.24 & 647.22 \\
\hline Jieyang & 585.99 & 724.23 & 816.09 & 1009.51 & 1225.86 \\
\hline Yunfu & 275.55 & 324.55 & 344.51 & 400.97 & 481.37 \\
\hline
\end{tabular}

Unit: Million Yuan

By applying matlab to engrave BP neural network, the results are shown in table 2. From the table, it is obvious that only the prediction values and target values of Foshan and Dongguan has large different, and the prediction of Guangzhou is more accurate.

Table 2: Prediction results of BP neural network

\begin{tabular}{|l|l|l|l|}
\hline Cities & $\begin{array}{l}\text { Actual Value in } \\
2011\end{array}$ & $\begin{array}{l}\text { Predicted Value in } \\
2011\end{array}$ & Error \\
\hline Guangzhou & 12423.439 & 12423.26613 & 0.17287165 \\
\hline Shenzhen & 11505.5298 & 10323.96187 & 1181.56793 \\
\hline Zhuhai & 1404.9305 & 1392.572804 & 12.35769552 \\
\hline Shantou & 1275.741206 & 1292.701673 & -16.96046704 \\
\hline Foshan & 6210.2348 & 11321.43458 & -5111.199776 \\
\hline Shaoguan & 816.8058 & 807.4783105 & 9.327489478 \\
\hline Heyuan & 579.2866 & 591.485381 & -12.19878104 \\
\hline Meizhou & 707.5366481 & 703.3016433 & 4.235004729 \\
\hline Huizhou & 2093.08078 & 2086.024747 & 7.056032295 \\
\hline Shanwei & 550.5542188 & 544.8147707 & 5.739448093 \\
\hline Dongguan & 4735.394887 & 7466.620282 & -2731.225395 \\
\hline Zhongshan & 2193.198524 & 2113.778865 & 79.41965816 \\
\hline Jiangmen & 1830.6373 & 1747.486858 & 83.15044207 \\
\hline Yangjiang & 766.8241 & 764.7073805 & 2.116719486 \\
\hline Zhanjiang & 1700.227167 & 1668.227266 & 31.99990104 \\
\hline Maoming & 1745.312951 & 1781.48796 & -36.17500903 \\
\hline Zhaoqing & 1324.414219 & 1342.759318 & -18.345099 \\
\hline Qingyuan & 1003.032566 & 1023.347019 & -20.31445363 \\
\hline Chaozhou & 647.2168419 & 623.0586055 & 24.15823641 \\
\hline Jieyang & 1225.8643 & 1201.178637 & 24.68566267 \\
\hline Yunfu & 481.367071 & 485.0645384 & -3.697467437 \\
\hline Unit: & & \\
\hline
\end{tabular}

Unit: Million

Yuan 


\section{Stability analysis of neural networks}

As shown in Figure 1, the hidden layer node number of neural network is 21, the input vector number is 4, which are the GDP from 2007 to 2010, and the target vector of output layer is the GDP in 2011. It is clear that neural network employ Leven-Marqardt training algorithm. Through the matlab programming, the result of iteration number is 82 steps. From Figure 2, where the weight circle part, namely when the iterative goes to the seventy-sixth step, it is realize the system has obtained the best confirmation value. At the same time, it also can meet the forecasting demand successfully.

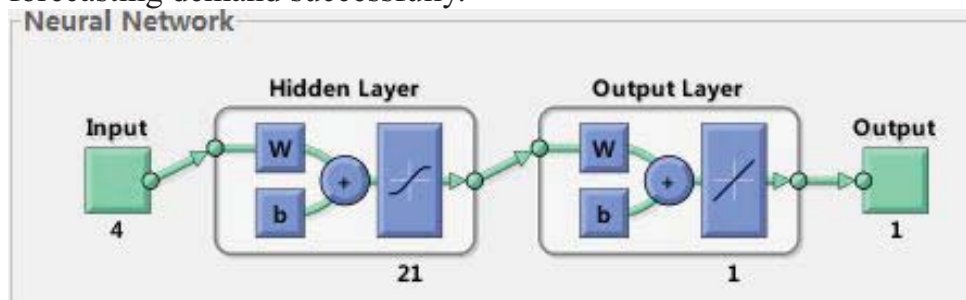

Figure 1: Neural network topology diagram

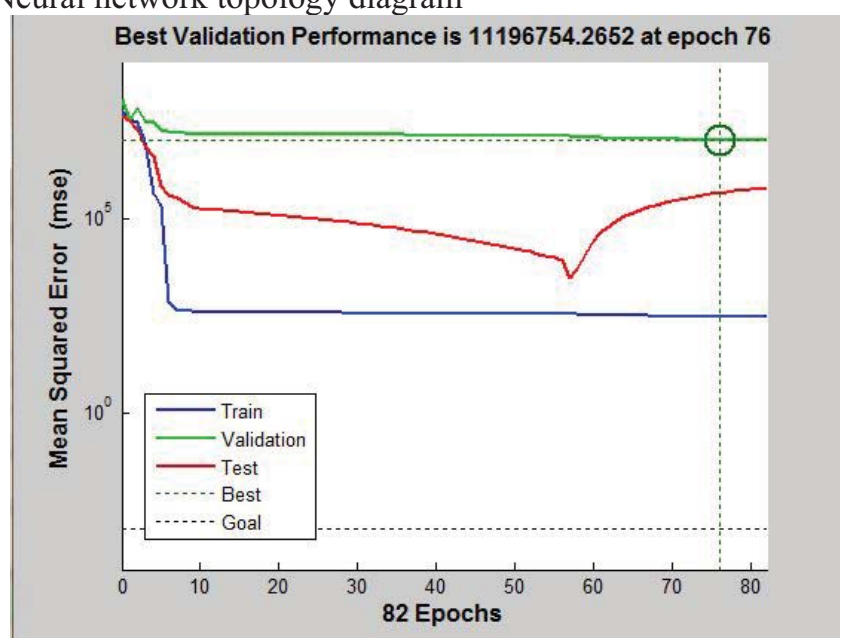

Figure 2: neural network iteration map

When the neural network iterative to the eighty-second step, where the iterative results gradient and variance has a good convergence. And the system confirmed the value is 6 , indicating that the neural network can predict effectively. The performance of the system is shown in Figure 3. 


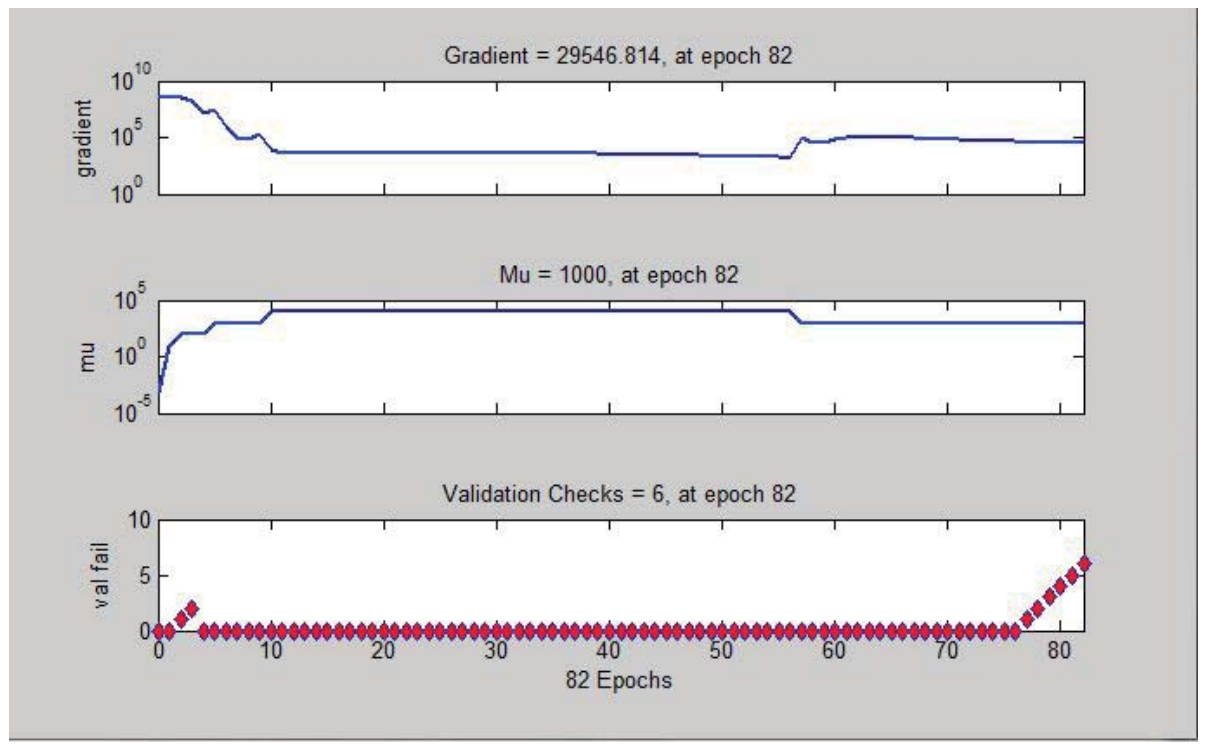

Figure 3: Gradient test map

Figure 4 demonstrates the results of neural network training. 


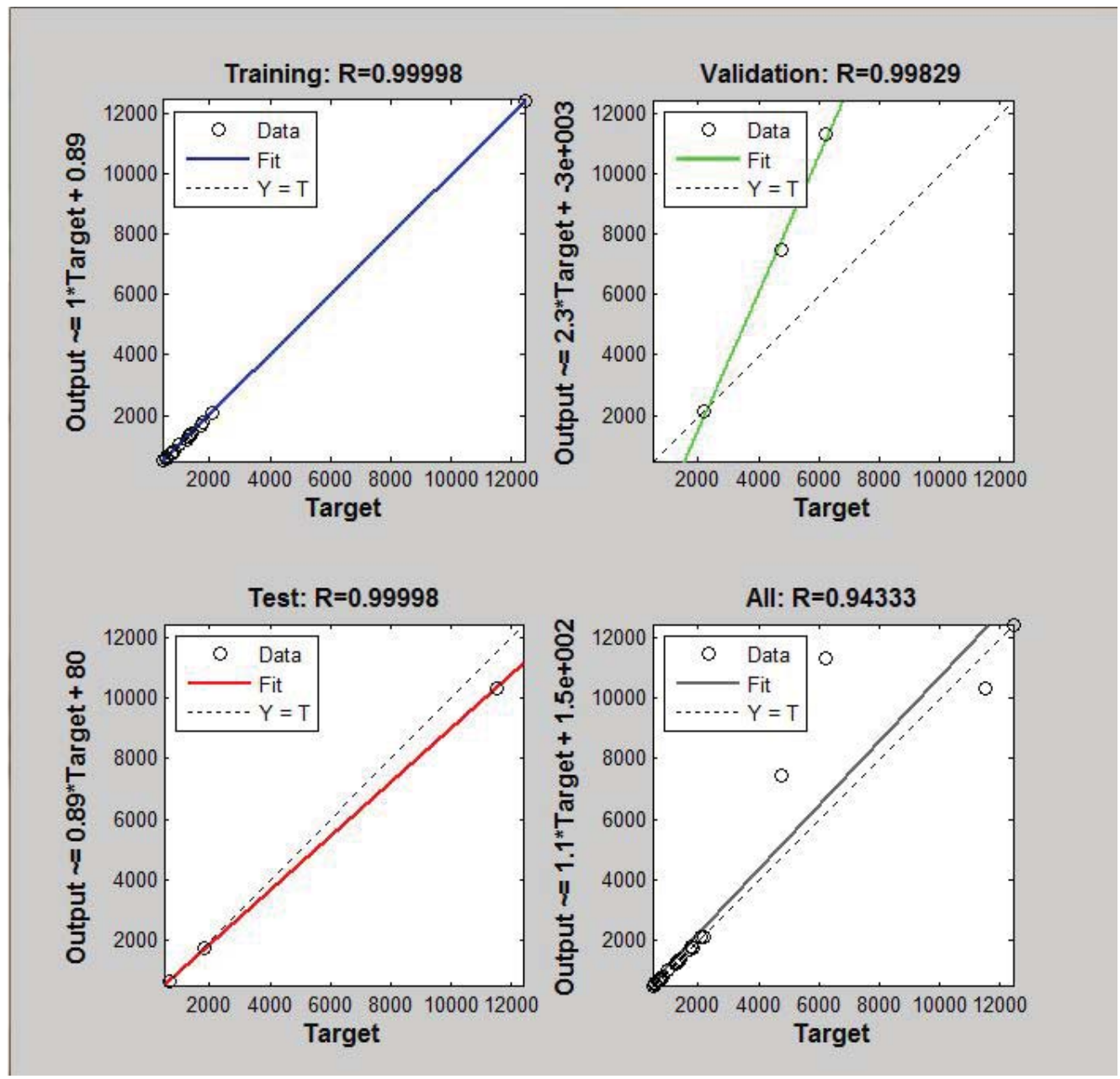

Figure 4: The results of training neural network map

Figure 3 shows that for the input vector, although the validity has deviation, in the test of the correction is great. Because of the GDP is relatively large, especially the difference in Pearl River Delta region and the northern areas. Therefore, the error comes up, which is a normal phenomenon. For the final result, only 2 values produce bigger deviation mentioned above, which are Dongguan and Shenzhen.

\section{Conclusion}

The GDP prediction model applies the self-learning characteristics of BP neural network, modifying the weights in the training process constantly, making the network actual output vector closer to the desired output, after that, analyzing the 
neural network weight matrix, utilizing the target vector to revise the weights and thresholds of the network frequently, and then getting the predicted values, which the error of the predicted value and the target vector are in an acceptable range. Therefore, it is going to draw a conclusion that is the establishment of GDP prediction model of BP neural network not only can make an accurate evaluation, but has a great potential development of BP neural network in forecasting GDP in the future.

\section{Acknowledgement}

This paper is financially supported by the Project (GD15XYJ09) of Guangdong Philosophy and Social Sciences and the youth project of Guangzhou Philosophy and Social Sciences.

\section{References}

[1] Wang Yongyi, Tu Jian. Neural network control [M]. Beijing: Mechanical Industry Press, 1999: 14-14

[2] Hechi- Nielsen R. Theory of t he back propagation neural net-work [J]. Proc of IJCNN, 1989(11): 593- 603

[3] Gross berg S A. Nonlinear neural network: Principles, machines and architectures [J]. Neural Net works, 1998(1): 15-57

[4] Yan Pingfan, Zhang Changshui, et al. Artificial neural network and evolutionary computing [M]. Beijing: Tsinghua University press, 2001: 17-24

[5] Cao Xingping, Yi Dongyun. Time series neural network prediction method based on $[\mathrm{J}]$. Advances in computer and information technology, 1999 (6): 1-3, 55

Name: Huang Rui

Date of birth (1981-09),

Male

Heyuan, Guangdong

Behavioral Finance and Regional Laboratory of Guangdong University of Finance,

Doctoral degree,

Assistant researcher,

Research on financial supervision and quantitative investment.

Name: Tang Zilong

Date of birth (1983-09),

Male

Changsha, Hunan

Behavioral Finance and Regional Laboratory of Guangdong University of Finance, 
Doctoral degree,

Lecturer

Research on financial supervision and financial model design

Name: Li Bin

Date of birth (1983-02),

Male

Huanghua, Hebei

Behavioral Finance and Regional Laboratory of Guangdong University of Finance,

Guangzhou Key Research Bases of Regional Financial Policy of Guangdong University of Finance

Doctoral candidate,

Vice-Professor

Research on Behavioral Finance and Regional Finance

Address: Guangzhou City, Tianhe District Ying Fu Road 527, Guangdong University of Finance, Behavioral Finance and Regional Laboratory

Cell phone: 13710726196

Zip code: 510521 